ISAHP Article: Cimler, Gavalec, Mls/ Optimal consistent approximation of a preference matrix To Be Submitted to the International Symposium of the Analytic Hierarchy Process 2014, Washington D.C., U.S.A.

\title{
OPTIMAL CONSISTENT APPROXIMATION OF A PREFERENCE MATRIX
}

\author{
Richard Cimler \\ Martin Gavalec \\ Karel Mls \\ Faculty of Informatics and Management \\ University of Hradec Kralove \\ Hradec Kralove, Czech Republic \\ E-mail: richard.cimler@uhk.cz
}

\begin{abstract}
Modern smartphones are powerfull devices with computing performance comparable to personal computers and laptops. Various sensors are embedded into these devices. These sensors are capable of monitoring a lot of different physical quantities which makes smartphones, together with smartphone computation performance, useful devices capable of monitoring and processing information about health status of person. It is possible to monitor position of person, not only wide area position using GPS but position of the body towards the earth surface as well. That enables to monitor the occurrence of critical situations such as a fall of the person to the ground. By using an accelerometer and a gyroscope it is also possible to monitor the person's breathing - more information can be found at (Suba, 2013). Other sensors can be connected to the smart phone by Bluetooth connection which enables monitoring further quantities such as the temperature on particular places on the body as well as of the environment.

There are several health applications collecting, processing, and transforming healthcare data into information, knowledge, and action. One of them is described in (Demirkan, H. 2013). Such health care applications need not only be small systems based on smart phone capabilities, but they also can be rather complex, see e.g. (Ou, Y.Y., 2013). Further devices can be located in the surrounding area and transmit information from sensors about the condition of the environment. Then the actuators controlled by the application adjust the environment in the location according the health status of the monitored person. Based on data from particular sensors, the application is expected to make a proposal of the action - to call an emergency, to be alert or to continue monitoring the situation. To meet these expectations, not only data from sensors, but expert knowledge is necessary, too, and multicriteria AHP model can merge these two contributions.

In AHP approach to decision making, priority scales are generally derived objectively after pair-wise subjective judgments are made. The construction and evaluation procedure of the AHP model can be summarized as follows (Saaty, 2008):

- Model the problem as a hierarchy containing the decision goal, the alternatives for reaching it, and the criteria for the alternatives' evaluation.

- $\quad$ Establish priorities among the elements of the hierarchy by making a series of judgments based on pairwise comparisons of the elements.

- $\quad$ Synthesize these judgments to yield a set of overall priorities for the hierarchy.

- $\quad$ Check the consistency of the judgments.

International Journal of the

1

Washington, D.C.

Analytic Hierarchy Process

June 29 - July 2, 2014
\end{abstract}


IJAHP Article: Cimler, Gavalec, Mls/ Optimal consistent approximation of a preference matrix To Be Submitted to the International Symposium of the Analytic Hierarchy Process 2014, Washington D.C., U.S.A.

- $\quad$ Reach the final decision based on the results of this process.

In AHP approach a $n \times n$ pair-wise comparison matrix $A$ with positive elements $a_{i j}$ is considered. Normalized principal eigenvector of the matrix $A$ is computed to get priorities of respective alternatives.

We say that $A$ is consistent, if $a_{i j} \bullet a_{j k}=a_{i k}$ for each $i, j, k$. If $a_{i j} \bullet a_{j k} \neq a_{i k}$ for some $i, j, k$, than $A$ is said to be inconsistent. The inconsistency of $A$ is measured by the consistency index $\mathrm{CI}_{n}$. To provide a measure independent of the order of the matrix $n$, (Saaty, 2009) proposed the consistency ratio (CR).

Watch Dog application is designed for Android mobile phones in order to gather information about the monitored person. System was primary focused on monitoring the persons with serious health issues. Due to the information from the accelerometer and the gyroscope it is possible to monitor the respiratory arrest, too.

All the information from the sensors has to be processed and the system evaluates the actual state of the monitored person according to the measured values. There are a lot of different important states of the person which can be recognized by the system. Some of the measured values can be normal, some of them can be suspicious and some can be strictly alerting. Some suspicious values can only signify danger when observed together with suspicious values from some other sensors. The importance of each sensor value can be different and various types of dangerous situations can be expressed by combination of measured values.

The experts helping to adjust the system are doctors - specialists on different medical sectors. There can be different opinions on the importance of the measured value combination. Each disease can have different set of symptoms, moreover every specialist has his specific view on the importance of the measured data. Thus the evaluation matrices from the experts could be inconsistent and contradicting. This situation has to be solved by suitable decision making approach.

The search for consensus in group decision making is typical in many real world situations. Several methods and tools, based on AHP were suggested to build consensus (Moreno-Jiménez, 2008). In medical applications, the need for more cautious approach to uniting of individual expert opinions is vital (Pecchia, 2013). To get the best evaluation of the model and to find the best alternative in the health monitoring application, not only consensus between experts, compromise or aggregation of individual judgments or priorities is sufficient. In our paper we propose to analyze inconsistency in individual expert evaluations and then to apply extremal algebra approach to construct the common evaluation of the decisional model.

Keywords: healthcare, AHP, sensors, extremal algebra, consistency.

\section{REFERENCES}

Demirkan, H. (2013). A Smart Healthcare Systems Framework: Sensors, Volume 15, Issue 5 , ISSN 1520-9202, WOS: 000325230700008.

Ou, Y.Y., Shih, P.Y., Chin, Y.H., Kuan, T.W., Wang, J.F., Shih, S.H. (2013). Framework of Ubiquitous Healthcare System Based on Cloud Computing for Elderly Living: 2013 Asia-Pacific signal and information processing association annual summit and conference (APSIPA), WOS: 000331094400195. 
IJAHP Article: Cimler, Gavalec, Mls/ Optimal consistent approximation of a preference matrix To Be Submitted to the International Symposium of the Analytic Hierarchy Process 2014, Washington D.C., U.S.A.

Pecchia, L., Martin, J.L., Ragoyyino, A., Vanyanella, C., Scognamiglio, A., Mirarchi, L., Morgan, S.P. (2013), User needs elicitation via analytic hierarchy process (AHP). A case study on a Computed Tomography (CT) scanner. BMC Medical Informatics and Decision Making 2013, 13:2 doi:10.1186/1472-6947-13-2.

Saaty, T.L. (2008). Decision Making for Leaders: The Analytic Hierarchy Process. RWS Publications, Pittsburgh.

Saaty, T.L. (2009). Theory and applications of the Analytic Network Process: Decision making with Benefits, Opportunities, Costs, and Risks. RWS Publications, Pittsburgh. Moreno-Jiménez, J.M., Aguarón, J., Escobar, M.T. (2008) The Core of Consistency in AHP-Group Decision Making. Group Decision and Negotiation, Volume 17, Issue 3, pp 249-265.

Suba, P, Tucnik, P. (2013). Mobile Monitoring System for Elder People Healthcare and AAL: Conference on Intelligent Environments, Volume 17, Pages 403 - 414 DOI: 10.3233/978-1-61499-286-8-403 